\title{
The clinical efficacy and safety of paclitaxel combined with avastin for NSCLC patients diagnosed with malignant pleural effusion
}

\author{
Zhen WAnG ${ }^{1 *} \odot$, Yisheng Zheng ${ }^{2}$, Zhenjian Fang ${ }^{3}$ \\ ${ }^{1}$ Department of Respiratory Medicine, Ningbo NO. 7 Hospital, Zhejiang, China \\ ${ }^{2}$ Department of Respiratory and Critical Care Medicine, Fuzong Clinical College of Fujian Medical University, Fuzhou General Hospital, Fujian, China \\ ${ }^{3}$ Department of Respiratory Medicine, Fuding Hospital, Fujian, China
}

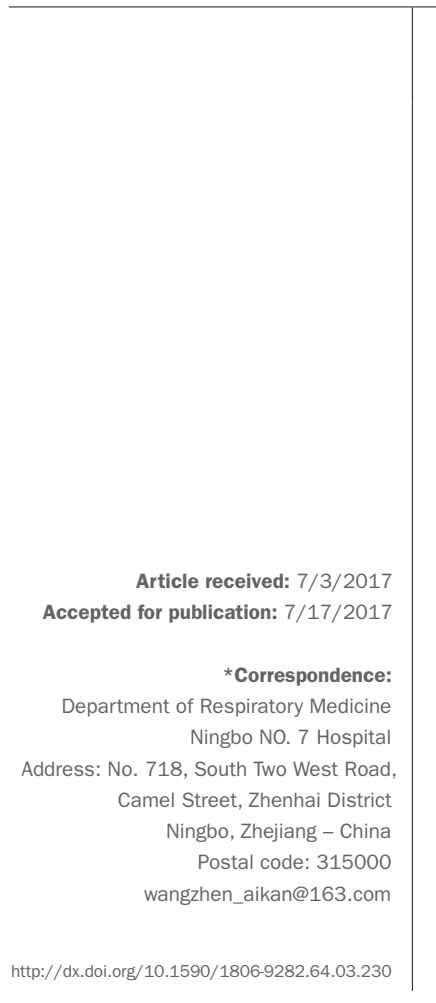

\section{SUMMARY}

Objective: The current study aimed to investigate the clinical efficacy of paclitaxel combined with avastin for non-small cell lung cancer (NSCLC) patients diagnosed with malignant pleural effusion (MPE).

Method: Total of 33 patients diagnosed with NSCLC as well as malignant pleural effusion were included. All of them received paclitaxel $(175 \mathrm{mg} / \mathrm{m} 2)$ and avastin $(5 \mathrm{mg} / \mathrm{kg}$ ). Clinical efficacy was evaluated using the total response rate, overall survival, progression-free survival and changes in MPE volume. Adverse events and rates of toxicities were examined as well.

Results: The total response rate reached $77 \%$ while the overall survival and the median progression-free survival were respectively 22.2 months and 8.4 months. Toxicities of grade 3-4 consisted of neutropenia in $57 \%$ of patients, anemia in $17 \%$ of them, febrile neutropenia in $11 \%$, as well as anorexia in $7 \%$. No treatment-correlated deaths were found.

Conclusion: Paclitaxel combined with avastin decreased MPE volume and increased survival rate of NSCLC patients via inhibiting vascular endothelial growth factor expression.

Keywords: Carcinoma, Non-Small-Cell Lung. Pleural Effusion, Malignant. Paclitaxel. Bevacizumab. Vascular Endothelial Growth Factor A.

\section{INTRODUCTION}

Lung cancer is found to be the major cause of cancer-correlated death in the most developed countries. Nearly $85 \%$ of lung cancer patients are diagnosed with nonsmall cell lung cancer (NSCLC) histology. ${ }^{1}$ Malignant pleural effusion (MPE) is one of the complications often found in patients with NSCLC. ${ }^{2-4}$ MPE treatment approaches include an indwelling pleural catheter (IPC), therapeutic thoracentesis and chemical pleurodesis. ${ }^{5}$ Avastin is a monoclonal antibody that can inhibit angiogenesis by inhibiting vascular endothelial growth factor (VEGF) expression, ${ }^{6}$ which is a tumor angiogenesis factor and participates in the development of pleural effusion. ${ }^{7}$ In the current study, we investigated the clinical efficacy of the traditional chemotherapy agent paclitaxel combined with avastin in the treatment of MPE. Our data suggested that paclitaxel combined with avastin was more effective to treat MPE.

\section{Method}

Patients and inclusion criteria

Total of 33 NSCLC patients diagnosed with MPE were recruited from January 2011 to December 2014. The inclusion criteria was (1) all patients were histopathologically diagnosed with adenocarcinoma at stages IV-M1a or IV-M1b in accordance with the International Association for the Study of Lung Cancer; ${ }^{8}(2)$ Karnofsky Performance Status $\geq 60 ; 9$ (3) MPE demonstrated by the identification of malignant cells in pleural fluid owing to metastases resulting from the tumors existing in the lung; (4) no abnormal findings on electrocardiography, bone marrow, liver and kidney function tests; (5) no allergic reaction to paclitaxel and avastin.

The study was approved by the Medical Ethics Committee of the West China Hospital (Chengdu, Sichuan, China). The study was conducted in accordance with the Declaration of Helsinki. Informed consent was provided by all subjects. 


\section{Treatment}

All patients were intra-pleurally injected with avastin $(5 \mathrm{mg} /$ $\mathrm{kg}$, Roche Diagnostics GmbH., Mannheim, Germany) in $100 \mathrm{~mL}$ of a solution followed by the same dose of paclitaxel once every three weeks for 12 consecutive weeks. A pigtail catheter (Suzhou Jingxin Medical Supplies Co., Ltd., Suzhou, China) was applied to the patients with MPEs for chest drainage and infusion of drugs. All procedures were B-ultrasound-guided and done at the bedside.

\section{Assessment of clinical efficacy and safety}

After baseline assessment, tumor lesions were assessed every four weeks during induction therapy and subsequent maintenance using computer tomography was performed every eight weeks until there was evidence of disease progression. Tumor response was evaluated based on version 1.1 of the Response Evaluation Criteria in Solid Tumor (RECIST) ${ }^{10}$ Toxicity was evaluated based on version 4.0 of the National Cancer Institute - Common Terminology Criteria for Adverse Events (NCI-CTCAE).

\section{Statistical analyses}

IBM SPSS version 17.0 was applied to conduct statistical analysis. Student's t test was used to compare the continuous variables. Pearson Chi-square or Fisher exact test were used to categorical variables. A multiple logistic regression analysis was conducted to evaluate possible predictors of poor prognosis. The area under the receiver operating characteristic curve (AUC) was calculated to investigate the ability of serum Hcy level to predict patient prognosis.

\section{ResULTS}

\section{Patient characteristics}

In all, 33 NSCLC patients diagnosed with MPE were recruited from January 2011 to December 2014. All patients were treated and evaluated for clinical efficacy and safety according to the study protocol. Baseline patient characteristics are summarized in Table 1 , as follows.

\begin{tabular}{|c|c|c|}
\hline Characteristics & $\mathbf{n}$ & $\%$ \\
\hline \multicolumn{3}{|l|}{ Age } \\
\hline Median range & \multicolumn{2}{|c|}{$65(31-77)$} \\
\hline \multicolumn{3}{|l|}{ Sex } \\
\hline Male & 24 & 73 \\
\hline Female & 9 & 27 \\
\hline \multicolumn{3}{|l|}{ ECOG PS } \\
\hline 0 & 19 & 58 \\
\hline 1 & 14 & 42 \\
\hline \multicolumn{3}{|l|}{ Stage } \\
\hline IIIB & 4 & 12 \\
\hline IV & 28 & 85 \\
\hline Relapse after surgery & 1 & 3 \\
\hline \multicolumn{3}{|l|}{ Histology } \\
\hline Adenocarcinoma & 31 & 94 \\
\hline Other & 2 & 6 \\
\hline \multicolumn{3}{|l|}{ EGFR gene mutation } \\
\hline Wild-type & 23 & 70 \\
\hline Mutated & 7 & 21 \\
\hline Not evaluated & 3 & 9 \\
\hline
\end{tabular}

Clinical efficacy of paclitaxel combined with avastin in the treatment of MPE

Treatment response of 33 patients was evaluated. Partial response was eventually found in 25 patients, and the ORR was 77\% (95CI 57-87\%) as shown in Table 2. Median TTR was 1.7 months (range $=0.6-5.8$ months), while the median progression-free survival (PFS) was 8.4 months (95CI 6.3-8.8 months), and the median OS was 22.2 months (95CI 13.8-28.1 months) (Figure 1).

Quality of life

EORTC QLQ-C30 includes five functional domains (physical, role, cognition, mood and social function), four

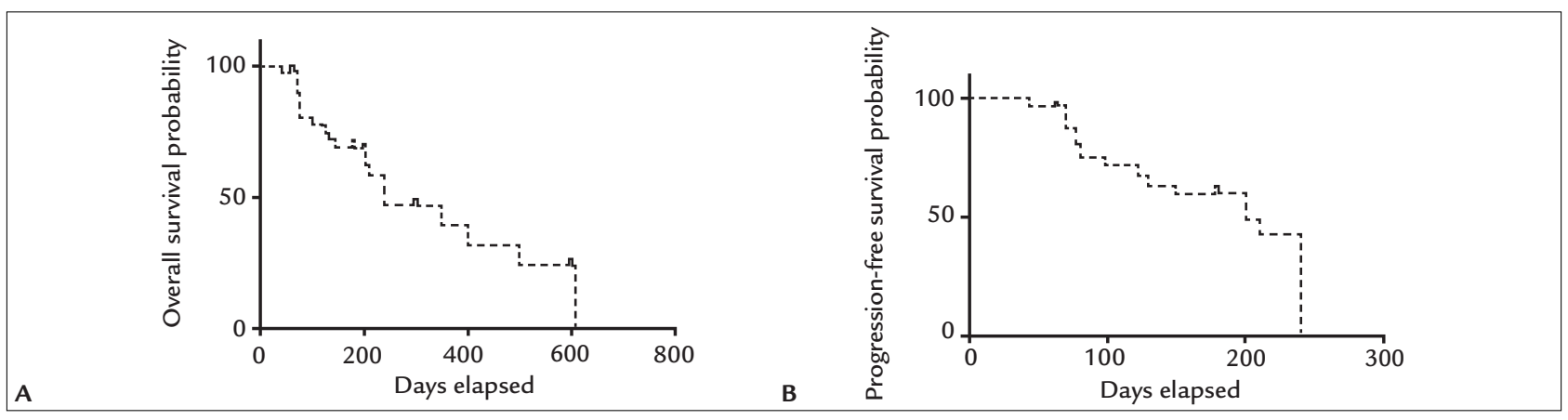

FIGURE 1 A. Overall survival (OS). B. Progression-free survival (PFS). 
TABLE 2 Treatment response.

\begin{tabular}{lll} 
Treatment & $\mathbf{n}$ & $\mathbf{\%}$ \\
\hline $\mathrm{CR}$ & 0 & \\
\hline $\mathrm{PR}$ & 25 & \\
\hline $\mathrm{SD}$ & 4 & \\
\hline $\mathrm{PD}$ & 3 & \\
\hline $\mathrm{NE}$ & 1 & 58 \\
\hline Total & 33 & 77 \\
\hline CR+PR & 25 & $57-87$ \\
\hline Response rate & & \\
\hline $95 \mathrm{Cl}$ & & $\mathrm{PD}$
\end{tabular}

CR: complete response; PR: partial response; SD: stable disease; PD: progressive disease.

symptoms (fatigue, pain, nausea and vomiting), one general health item and five common single entry symptoms (dyspnea, loss of appetite, insomnia, constipation, and diarrhea). Calculate standardized scores for each field (SS). Results showed that overall health improved after treatment, and dyspnea improved most during symptom assessment (Table 3).

\section{Toxicity}

The adverse events found in the total of 33 patients are listed below in Table 4 . Hematological toxicities reaching grade $3 / 4$ were neutropenia (18 patients, $55 \%$ ), leukopenia (seven patients, 21\%), anemia (six patients, 18\%), thrombocytopenia (one patient, $3 \%$ ) and febrile neutropenia (four patients, $12 \%$ ). Non-hematological toxicities reaching grade $3 / 4$ were anorexia (three patients, $9 \%$ ), diarrhea (one patient, 3\%) and aminotransferase elevation (one patient, $3 \%$ ). The most common hemorrhage was nasal bleeding, which occurred in 14 patients (42\%). Gingival bleeding appeared in two patients (6\%), and hemorrhoid bleeding appeared in one (3\%). All the cases of hemorrhage were grade 1 . It is worth noting that only three patients (9\%) had grade 2 neuropathy, and none of the patients had grade $3 / 4$ neuropathy. One had grade 1 pneumothorax and two patients had hyperkalemia (grade 2 and grade 3 ). No treatment-related deaths were observed.

TABLE 3 Changes in mood, general health and dyspnea before and after treatment $(x \pm s)$.

\begin{tabular}{llll} 
& Mood & $\begin{array}{l}\text { General } \\
\text { health }\end{array}$ & Dyspnea \\
\hline Before treatment & $48.3 \pm 6.5$ & $37.6 \pm 5.2$ & $80.1 \pm 3.9$ \\
\hline After treatment & $62.8 \pm 4.3$ & $54.2 \pm 6.4$ & $51.5 \pm 3.6$ \\
\hline
\end{tabular}

TABLE 4 Summary of adverse events.

Grade (NCl-CTCAE) Grade

\begin{tabular}{llllll}
\hline 0 & 1 & 2 & 3 & 4 & $3 / 4$
\end{tabular}$(\%)$

\begin{tabular}{|c|c|c|c|c|c|c|}
\hline Hematological toxi & & & & & & \\
\hline Leukopenia & 5 & 6 & 15 & 7 & 0 & 21 \\
\hline Neutropenia & 3 & 3 & 9 & 15 & 3 & 55 \\
\hline Anemia & 0 & 14 & 13 & 6 & 0 & 18 \\
\hline Thrombocytopenia & 9 & 19 & 4 & 1 & 0 & 3 \\
\hline Febrile neutropenia & 29 & 0 & 0 & 4 & 0 & 12 \\
\hline Non-hematological & & & & & & \\
\hline Anorexia & 13 & 15 & 2 & 3 & 0 & 9 \\
\hline Nausea & 16 & 13 & 4 & 0 & 0 & 0 \\
\hline Vomiting & 25 & 6 & 2 & 0 & 0 & 0 \\
\hline Diarrhea & 26 & 4 & 2 & 1 & 0 & 3 \\
\hline Constipation & 16 & 15 & 2 & 0 & 0 & 0 \\
\hline Fatigue & 16 & 13 & 4 & 0 & 0 & 0 \\
\hline Infection & 31 & 0 & 2 & 0 & 0 & 0 \\
\hline Alopecia & 9 & 13 & 11 & 0 & 0 & 0 \\
\hline Neuropathy & 11 & 19 & 3 & 0 & 0 & 0 \\
\hline Hypertension & 30 & 2 & 1 & 0 & 0 & 0 \\
\hline Nasal bleeding & 19 & 14 & 0 & 0 & 0 & 0 \\
\hline Other & 30 & 3 & 0 & 0 & 0 & 0 \\
\hline Proteinuria & 26 & 2 & 5 & 0 & 0 & 0 \\
\hline AST/ALT & 16 & 14 & 2 & 1 & 0 & 3 \\
\hline Total bilirubin & 24 & 7 & 2 & 0 & 0 & 0 \\
\hline Creatinine & 28 & 4 & 1 & 0 & 0 & 0 \\
\hline
\end{tabular}

NCI-CTCAE: National Cancer Institute - Common Terminology Criteria for Adverse Events; AST/ALT: aspartate transaminase/alanine aminotransferase.

\section{Discussion}

The present study aimed to explore optimization therapy in treatment of MPE. The results have demonstrated that paclitaxel combined with avastin resulted in high ORR with good tolerance. These results suggested that use of avastin as a VEGF inhibitor resulted in an additive beneficial effects in treatment of MPE. In clinical practice, first-line treatment for MPE includes chemotherapy aimed at reducing pleural fluid volume. Nevertheless, it has been found that high levels of VEGF contribute to angiogenesis and serous cavity effusions in cancer patients, ${ }^{9}$ and the occurrence of MPE is related to increased expression of VEGF receptor in lung cancer cells of human beings. ${ }^{11}$ Avastin is a monoclonal antibody against VEGF and has been applied to treat NSCLC in clinical practice. ${ }^{12,13}$ Therefore, it is reasonable to hypothesize that clinical efficacy of avastin in treatment of MPE was related to suppressing angiogenesis via inhibiting VEGF expression. ${ }^{14-16}$ 
In the present study, adverse events of the drugs were recorded according to the CTCAE v3.0. ${ }^{17}$ The results have shown that most patients had side effects ranked from grade 1 to 2. Paclitaxel has an essential clinical activity in fighting against a wide range of tumor types such as lung cancer. ${ }^{18}$

The antineoplastic agent interferes with the growth of both cancer cells and normal body cells, which are eventually destroyed with occurrence of some unwanted effects. ${ }^{19,20}$ Since these observed side effects were common and not serious for the patients taking paclitaxel, it is conceivable that intervention with avastin not only intensified the treatment effect of the anticancer drug in the patients but also shortened hospitalization time, reducing hospital costs.

\section{Conclusion}

We investigated the clinical efficacy of avastin along with paclitaxel in the treatment of MPE in patients diagnosed with NSCLC. The results demonstrated that avastin combined with paclitaxel was effective and safe in terms of improving treatment success and survival rates.

\section{References}

1. Herbst RS, Heymach JV, Lippman SM. Lung cancer. N Engl J Med. 2008;359(13):1367-80.

2. Fenton KN, Richardson JD. Diagnosis and management of malignant pleural effusions. Am J Surg. 1995;170(1):69-74.

3. Antony VB, Loddenkemper R, Astoul P, Boutin C, Goldstraw P, Hott J, et al. Management of malignant pleural effusions. Eur Respir J. 2001;18(2):402-19.

4. Johnston WW. The malignant pleural effusion. A review of cytopathologic diagnoses of 584 specimens from 472 consecutive patients. Cancer. 1985;56(4):905-9.
5. Musani AI. Treatment options for malignant pleural effusion. Curr Opin Pulm Med. 2009;15(4):380-7.

6. Reeder LB. Malignant pleural effusions. Curr Treat Options Oncol 2001;2(1):93-6.

7. Irani DR, Underwood RD, Johnson EH, Greenberg SD. Malignant pleural effusions. A clinical cytopathologic study. Arch Intern Med. 1987; 147(6):1133-6.

8. Rusch VW, Figlin R, Godwin D, Piantadosi S. Intrapleural cisplatin and cytarabine in the management of malignant pleural effusions: a Lung Cancer Study Group trial. J Clin Oncol. 1991;9(2):313-9.

9. Schag CC, Heinrich RL, Ganz PA. Karnofsky performance status revisited: reliability, validity, and guidelines. J Clin Oncol. 1984;2(3):187-93.

10. Eisenhauer EA, Therasse P, Bogaerts J, Schwartz LH, Sargent D, Ford R, et al. New response evaluation criteria in solid tumours: revised RECIST guideline (version 1.1). Eur J Cancer. 2009;45(2):228-47.

11. Murphy WK, Fossella FV, Winn RJ, Shin DM, Hynes HE, Gross HM, et al. Phase II study of taxol in patients with untreated advanced non-small-cell lung cancer. J Natl Cancer Inst. 1993;85(5):384-8.

12. Oken MM, Creech RH, Tormey DC, Horton J, Davis TE, McFadden ET, et al. Toxicity and response criteria of the Eastern Cooperative Oncology Group. Am J Clin Oncol. 1982;5(6):649-55.

13. Das M, Wakelee H. Targeting VEGF in lung cancer. Expert Opin Ther Targets. 2012;16(4):395-406.

14. Pallis AG, Syrigos KN. Targeting tumor neovasculature in non-small-cell lung cancer. Crit Rev Oncol Hematol. 2013;86(2):130-42.

15. Hsu IL, Su WC, Yan JJ, Chang JM, Lai WW. Angiogenetic biomarkers in nonsmall cell lung cancer with malignant pleural effusion: correlations with patient survival and pleural effusion control. Lung Cancer. 2009;65(3):371-6.

16. Petrou M, Kaplan D, Goldstraw P. Management of recurrent malignant pleural effusions. The complementary role of talc pleurodesis and pleuroperitoneal shunting. Cancer. 1995;75(3):801-5.

17. Robinson LA, Fleming WH, Galbraith TA. Intrapleural doxycycline control of malignant pleural effusions. Ann Thorac Surg. 1993;55(5):1115-21.

18. Trotti A, Colevas AD, Setser A, Rusch V, Jaques D, Budach V, et al. CTCAE v3.0: development of a comprehensive grading system for the adverse effects of cancer treatment. Semin Radiat Oncol. 2003;13(3):176-81.

19. Monsarrat B, Chatelut E, Royer I, Alvinerie P, Dubois J, Dezeuse A, et al. Modification of paclitaxel metabolism in a cancer patient by induction of cytochrome P450 3A4. Drug Metab Dispos. 1998;26(3):229-33.

20. Chang AY, Kim K, Glick J, Anderson T, Karp D, Johnson D. Phase II study of taxol, merbarone, and piroxantrone in stage IV non-small-cell lung cancer: The Eastern Cooperative Oncology Group Results. J Natl Cancer Inst. 1993;85(5):388-94. 